\title{
Example of a group action determined phase transition
}

Jeffrey W. Felix

Dorian M. Hatch

hatchd@byu.edu

Follow this and additional works at: https://scholarsarchive.byu.edu/facpub

Part of the Astrophysics and Astronomy Commons, and the Physics Commons

\section{Original Publication Citation}

Felix, J. W. and D. M. Hatch."Example of a group action determined phase transition." The Journal of Chemical Physics 82 (1985): 1496-153.

\section{BYU ScholarsArchive Citation}

Felix, Jeffrey W. and Hatch, Dorian M., "Example of a group action determined phase transition" (1985). Faculty Publications. 756.

https://scholarsarchive.byu.edu/facpub/756 


\title{
Example of a group action determined phase transition
}

\author{
J. W. Felix and D. M. Hatch \\ Brigham Young University, Provo, Utah 84602
}

(Received 16 August 1984; accepted 11 October 1984)

\begin{abstract}
The principles of the group action approach to structural phase transitions are outlined. It is assumed that all properties of the transition are determined by the action of a single physically irreducible represention of the space group of the more symmetric phase. We determine the isotropy groups using the image space of the representation. The free energy minima are determined to fourth order and to all orders using the results of Gufan and then compared. This theory is applied to Calcite $(R \overline{3} c)$ to determine all possible continuous commensurate phase transitions.
\end{abstract}

\section{INTRODUCTION}

An assumption, not always explicitly stated, of the basic Landau or mean field theory of continuous phase transitions (CPTs), is that of physical irreducibility or irreducibility on the real.' That is, all properties of the transition are determined by an order parameter which transforms irreducibly over the real numbers under the symmetry group of the high symmetry phase (equivalent to the use of coreps and grey groups.) $)^{2}$ As Dimmock ${ }^{3}$ pointed out, this assumption ignores mode-mode interactions which are certainly present even in mean field theory.

It was noted in Ref. 2 that the proper way to deal with these mode-mode interactions is by constructing a suitable linear combination of mode amplitudes and treating the effective Hamiltonian of the degrees of freedom so obtained with the renormalization group (RG). One can also take this effective Hamiltonian as the mean field free energy, but in general this yields a very intractable problem. One must solve a set of nonlinear partial differential equations to find extrema.

If one is interested only in studying the appearance of commensurate ordering, however, the assumptions of the Landau theory have been found to yield the listing of ordering phases, including phases reached by first order transitions from the prototypic phase. Indeed, it can be shown that if one is willing to consider a Landau free energy polynomial of sufficiently high order in the order parameter, one will find all possible commensurate ordering phases of the system among the minima of the free energy ${ }^{4}$ for some range of the coefficients.

However, it is less successful at predicting the nature of the phase lines, e.g., first-order vs continuous, or critical exponents. One thing, at least, seems clear in this regard. If Landau theory predicts a first order phase transition, the RG will not change this prediction. ${ }^{5}$

In the past few years, from the basic Landau theory, a coherent and well-formulated theory for dealing with symmetry breaking effects in a structural phase transition in the irreducible case has developed. ${ }^{6}$ The irreducibility of the order parameter components allows one to employ a great many algebraic methods in carrying out this complete minimization, at least as far as determining the basic nature of the various phases, and predicting firstorder transitions. Indeed, using the full algebraic power of these methods, one can simplify the calculations considerably.

We here will briefly outline the current state of the theory and in an example, calcite, which has prototype $R \overline{3} c\left(D_{3 d}^{6}\right)$ symmetry, calculate the phase diagram. Calcite, $\mathrm{CaCO}_{3}(\mathrm{I})$, possessing a trigonal space group symmetry, and a structural phase transition at elevated pressure, has been studied recently both theoretically and experimentally as a system likely to show unique critical solid-solid behavior. $^{7}$

\section{BASIC THEORY}

The Landau theory, then, assumes the existence of a physical field, tensor or otherwise, which, in the vicinity of the phase transition from the prototypic phase, can be expanded in terms of a number of ordering thermodynamic variables, called variously order or transition parameters. $^{8}$

For calcite, which undergoes a structural phase transition, the physical tensor can be chosen as the particle density and the order parameter as the collection of degenerate mode amplitudes of a zone boundary soft mode. Thus we write the density as

$$
\rho(\mathbf{r})=\rho_{0}(\mathbf{r})+\sigma_{i} \Psi_{i}(\mathbf{r}),
$$

where

$$
g \Psi_{i}(\mathbf{r})=\Psi_{j}(\mathbf{r}) D_{j i}(g),
$$

for $g \in G$, the space group which leaves $\rho_{0}(\mathbf{r})$ invariant. The $\sigma_{i}$ are the order parameter components. Here we employ a summation convention, with repeated indices being summed from 1 to $n, n$ being the dimension of the physically irreducible representation $D(G) . \sigma$ resides in a space $C$, the carrier space of $D$, of dimension $n$.

Since $D(G)$ is a homomorphism of $G$, by definition, we can use the fact that homomorphisms preserve all algebraic relationships. Thus all consequences of the symmetry of the problem follow from the properties of 
$D(G)$ acting on $C$, the group action of $G$ on $C$. Thus, for example subgroups of $G$ will be mapped onto subgroups of $D(G)$, and vice versa, if one refers to the inverse image mapping, and the properties of the subgroups of $G$ are determined by those of $D(G)$. In the following $D(G)$ will also be denoted by $L$, and be referred to as the image group of $G$ under $D$, or more simply, just the image group.

For commensurate phase transitions, $L$ will always be a finite group. Furthermore, since it acts irreducibly on the real on $C$, it will always be isomorphic to a point group in $n$ dimensions. Indeed, it will always be isomorphic to a point group in $n$ dimensions which has an $n$ dimensional faithful representation. This last observation greatly reduces the number of representative systems one needs to study. For example, when $n=3$, there are only five possible image groups. ${ }^{9}$

For $n \leqslant 3$ all of these image groups have been studied and a complete Landau theory analysis has been carried out. ${ }^{9}$ For irreps, then, with $n \leqslant 3$ we need only specify the isomorphism from the standard results to $D(G)$ to obtain complete information. All of the irreps of $R \overline{3} c$ of interest in calcite happen to fall in this category.

To actually determine the physical states, or possible phases, we construct a general free energy $F(\sigma)$ with appropriate symmetry, and minimize it. Suppose $F(\sigma)$ obtains an absolute minimum at $\sigma=\sigma^{\prime}$. In the ordered phase some largest subgroup of $G$ will leave $\rho(r)$ in Eq. (1) invariant. Such a subgroup of $G$ is known as an isotropy group of $G$ with respect to $D$. One now invokes the Curie Principle ${ }^{10}$

The symmetry group of a physical system is the intersection of the symmetry elements of all its physical properties.

It then follows that, since the Hamiltonian is still invariant under $G$, the symmetry group of the system, in the absence of other determining properties and when $\sigma^{\prime}$ is nonzero, is the largest subgroup of $G$ leaving $\sigma^{\prime}$ invariant, i.e., the isotropy group of $\sigma$.

It can be shown that, if one keeps enough terms in the expansion of $F(\sigma)$, all points of the carrier space of $D$, correspond to extrema of $F$ for some particular value of the expansion parameters. ${ }^{11}$ This, in turn, implies that in the set of all extrema of $F$, specified by $\partial F / \partial \sigma_{i}=0$, one obtains all isotropy subgroups of $G$. Thus, one way to obtain possible candidates for minima is to list all such isotropy subgroups and their associated $\sigma^{\prime}$. Indeed, one does not usually distinguish as distinct phases different $\sigma^{\prime}$ with the same isotropy group or conjugate isotropy groups, since they correspond to different domains of the same phase, at possibly different temperature. This leads to the ideas of orbits and strata. ${ }^{12}$

Given a particular $\sigma$, its orbit is the set of all $D(g) \sigma$ as $g$ ranges over $G$. Clearly, the isotropy groups of different points in the orbit are conjugate. The collection of all points in our carrier space with equivalent isotropy groups, or equivalently the collection of all orbits of the same type form a stratum. The collection of all strata fills out $C$. It is clear that points in the same stratum correspond to the same phase, albeit in perhaps different domains, or at different field points $\mathbf{f},(\mathbf{f}=T, P, \mathbf{H}$, etc.). This is because the essential characteristic of each phase is its symmetry group. We then list all strata and see which actually minimize the free energy at a given field point. ${ }^{6}$

A method developed by Birman and Jaric ${ }^{13}$ allows one to find all such strata or equivalently all isotropy groups and has been shown to be equivalent to the use of the Curie principle in finding allowed isotropy groups. ${ }^{13}$ The method lists subgroups of $G$ in subgroup chains, i.e., $G \supset G_{1} \supset G_{2} \supset \cdots \supset G_{n}$. Then one calculates the subduction frequencies

$$
i\left(G_{j}\right)=\underset{G_{j}}{M} \chi(g),
$$

which is the number of times the identity rep of $G_{j}$ appears in the representation of $G_{j}$ subduced by $D(g)$. (Here

$$
\underset{G_{j}}{M}=\frac{1}{\left|G_{j}\right|} \sum_{g \in G_{j}},
$$

is the group mean over $H$.) $i\left(G_{j}\right)$ is also the number of invariant vectors in the space of $\sigma$ under the action of $G_{j}$. If the number of such invariant, linearly independent vectors is the same under the action of a isotropy subgroup of $G_{j}$, e.g., $G_{m}$ then by the Curie Principle we select $G_{j}$ as the meaningful isotropy group. This then is the Chain Subduction Criterion. ${ }^{13}$

If $G \supset G_{1} \supset G_{2}$ and $i\left(G_{1}\right)=i\left(G_{2}\right)$ then $G_{2}$ is not an isotropy group of the associated representation.

One also says $G_{2}$ is disallowed. Noting, that when $G_{1}$ $\supset G_{2}$ necessarily $i\left(G_{i}\right) \leqslant i\left(G_{2}\right)$, in the case when $i\left(G_{1}\right)$ $<i\left(G_{2}\right)$ then $G_{2}$ is allowed and corresponds to a distinct stratum.

It can be shown further that in searching for possible allowed isotropy subgroups of $G$ one need only search among the inverse images of image subgroups. The subgroups of all such inverse image are then disallowed by the chain subduction criterion. Thus, by finding all isotropy subgroups of $L=D(G)$, one then obtains all isotropy subgroups of $G$ by taking inverse images, i.e., $i\left(L^{\prime}\right)=i[D(H)]=i(H)$ where $H=D^{-1}\left(L^{\prime}\right)$, the largest subgroup of $G$ mapped onto $L^{\prime}$. That only these subgroups of $G$ occur as isotropy subgroups follows from the fact that all $H \subset G$ are inevitably mapped onto some $L^{\prime}$ $=D(H)$ and clearly $H \subset D^{-1}\left(L^{\prime}\right)$. Thus all allowed isotropy groups will of necessity contain the kernel of the representation, $\operatorname{ker}_{D} G$, i.e., the collection of symmetry elements which are mapped onto the identity matrix of the representation. This also follows from the fact that the generic stratum, or most general point of $C$, has isotropy group $\operatorname{ker}_{D} G$.

Since the image group (irrep) is real and orthogonal it must be isomorphic to a finite subgroup of $O(n)$, i.e., a point group. If the representation is of dimension three or less it is always isomorphic to one of the 32 crystal point groups, whose subgroup structure is well documented. ${ }^{14}$ When $n$ is greater than three, it is most convenient to work with the group elements themselves 
and their composition law, removing symmetry elements in a systematic fashion and checking the group properties. ${ }^{15-17}$

Since in the vicinity of the transition $\sigma$ is small, we assume that the free energy can be written as

$$
F=F(\sigma)=\sum_{m=0}^{\infty} \frac{1}{m !} F_{l_{m}}^{m} \sigma_{i_{1}} \cdots \sigma_{i_{m}},
$$

where $F$ must be invariant when $\sigma$ is replaced by $D(g) \sigma$, $g$ an element of the space group of the system $G . F$, of course, must also be real.

Now, clearly, order by order, $F(\sigma)$ consists of symmetric polynomial invariants in the components of $\sigma$. The set of all polynomial invariants in $\sigma$ form an algebra over the real numbers. Well established algebraic theo$\mathrm{rems}^{18}$ then state that for finitely generated groups (referring here to the image of $D$ ), there are a finite number (actually $n=\operatorname{dim} D$ for a finite group) of algebraically independent invariant polynomials (invariants) $I_{j}(\sigma), j$ $=1$ to $n$ and relatively invariant polynomials (relative invariants) $J_{j}(\sigma), j=1$ to $s$, such that $J_{j}=F_{j}(I)$ for some polynomial $F_{j}$. Further, all analytic invariants, in particular $F(\sigma)$, can be written

$$
F(\sigma)=F(\mathbf{I}, \mathbf{J})=G(\mathbf{I})+\sum_{j} J_{j} H(\mathbf{I})
$$

The number and degree of each kind of polynomial can be determined with the use of the Molien function, defined as

$$
M(t)=\underset{g}{M} \operatorname{det}\left[(I-t D(g)]^{-1} .\right.
$$

$M(t)$ can always be written ${ }^{18}$

$$
\begin{aligned}
& M(t)=\frac{N(t)}{D(t)}, \quad D(t)=\prod_{i=1}^{n}\left(1-t^{d_{i}}\right), \\
& N(t)=\sum_{\alpha=0}^{\nu-1} t^{\delta_{\alpha}}, \quad \delta_{0}=0
\end{aligned}
$$

where $d_{i}$ is the degree of $I_{i}(\sigma)$ and $\delta_{\alpha}$ is the degree of $J_{\alpha}(\sigma)$. As noted before, when the $D$ is of small dimension, it will be isomorphic to a crystal point group, for which the Molien functions and invariant bases have been tabulated in the literature. ${ }^{19,20}$

Using Eq. (5) one can now find all possible extrema. This is typically done in one of two ways. One can find the isotropy subgroups of $G$, and then project out the extrema of $F(\sigma)$, or first find the extrema directly and then find the isotropy groups from them. We will carry out the first step of the first approach and also complete the second for the particular case of $R \overline{3} c$.

In addition to the above requirements for a continuous phase transition we mention three more. The first is the Lifshitz condition, ${ }^{1}$ which actually arises from a more general mean field theory, as well as following from the renormalization group. It is a necessary condition for the transition to be commensurate, namely

$$
\{D\}^{2} \times V \text { must not contain the identity rep. }
$$

$V$ is here the vector representation of $G$. In terms of characters this becomes

$$
\underset{g}{M}\{\chi\}^{2}(g) \phi(g)=0,
$$

where $\chi(g)=\operatorname{tr} D(g)$ and $\phi(g)=\operatorname{tr} V(g)$. The second is the Landau condition ${ }^{1}$ which in group-theoretical terms may be stated as

$[D]^{3}$ must not contain the identity rep.

In terms of characters this can be written,

$$
\underset{g}{M}[\chi]^{3}(g)=0 .
$$

A third condition, implicit in our previous discussion, is that the order parameter should correspond to a physical tensor field, ${ }^{21}$ or in the development given here, that $D(g)$ should actually appear in the list of reps reducing $u$, the displacement field. Here $u$ is the collection of all displacement vectors at all lattice cells at $\mathbf{R}$ and cell basis vectors $d$. Then $g u=u \Gamma^{d}(g)$, where the displacement field representation is $\Gamma^{d}$. This condition may be stated as

$$
\Gamma^{d} \text { must subduce } D \text { at least once, }
$$

or in terms of characters,

$$
f=\underset{g}{M} \chi^{d}(g) \chi(g)>0 .
$$

Note that $\Gamma^{d}$ is real. Using the induced nature of $D(G)$ and the special structure of $D^{d}$, this can be written in a considerably simplified form, ${ }^{22}$ i.e., the frequency of $\mathrm{Eq}$. (10),

$$
\begin{aligned}
f_{j}^{(\mathbf{k})}= & c_{j} \underset{\bar{K}}{M} \chi_{j}^{\mathbf{k}}(S \mid \tau) \phi(S) \sum_{d} e^{-i \mathbf{k} \cdot \mathbf{R}_{d}(S)} \\
& \times \delta\left[\mathbf{d}-S^{-1}(\mathbf{d}-\tau)+R_{d}(S)\right],
\end{aligned}
$$

must be positive, where $\chi_{j}^{(\mathbf{k})}(S \mid \mathbf{t})$ is a character of the space group element $(S \mid t)$ in the $j$ th small rep of $K$, the little group of $\mathbf{k}, \bar{K}$ being the point group of $\mathbf{k} . c_{j}$ is 1 or 2 depending on whether $D$ is irreducible or irreducible on the real only, and $\mathbf{R}_{d}(S)=S^{-1}(\mathbf{d}-\tau)-\mathbf{d}^{\prime}$, where (R, d) $\rightarrow\left(R^{\prime}, d^{\prime}\right)$ under the space group element $(S \mid \mathbf{t}+\tau)$.

Equations (8) and (9), and (8) in particular, greatly reduce the number of reps, or irreps that need to be considered as possible driving irreps for a CPT. It can be shown that the only irreps which satisfy this condition correspond to points of symmetry of the holosymmetric point group in the first Brillouin zone, which are few in number. ${ }^{3}$ The new symmetry group leaves $\sigma^{\prime}$ invariant and is a subgroup of $G$.

Once one has the free energy form, and has found the extrema, either through direct calculation or projection techniques using isotropy groups one checks to see if they can be minima. It is likely that if $F(\sigma)$ is expanded to high enough degree any extrema can be made a minima for some value of the expansion parameters. In many cases, e.g., in the RG, only terms up to a certain degree enter in, in which case this might not always be possible. In any event some of the minima will still not lead to continuous transitions unless, as $\sigma \rightarrow 0$, all conditions on the parameters necessary for real, minimal solutions can be maintained. In general this is not possible and so only 
certain extrema lead to possible continuous phase transitions. This is demonstrated explicitly for the image groups when $n$ is less than three in Ref. 9.

\section{APPLICATION TO CALCITE}

The space group $R \overline{3} c$ is based on a trigonal lattice with the maximal or holosymmetric trigonal point group $\overline{3} m\left(D_{3 d}\right)$. If $a, c$ are the lattice constants then we chose the lattice basis vectors as

$$
\begin{aligned}
& \mathbf{t}_{1}=-a \hat{j}+c \hat{k}, \\
& \mathbf{t}_{2}=\frac{\sqrt{3}}{2} a \hat{i}+\frac{1}{2} a \hat{j}+c \hat{k}, \\
& \mathbf{t}_{3}=-\frac{\sqrt{3}}{2} a \hat{i}+\frac{1}{2} a \hat{j}+c \hat{k},
\end{aligned}
$$

which yield the reciprocal lattice basis vectors

$$
\begin{aligned}
& \mathbf{g}_{1}=2 \pi\left(-\frac{2}{3 a} \hat{k}_{y}+\frac{1}{3 c} \hat{k}_{z}\right), \\
& \mathbf{g}_{2}=2 \pi\left(\frac{1}{a \sqrt{3}} \hat{k}_{x}+\frac{1}{3 a} \hat{k}_{y}+\frac{1}{3 c} \hat{k}_{z}\right), \\
& \mathbf{g}_{3}=2 \pi\left(-\frac{1}{a \sqrt{3}} \hat{k}_{x}+\frac{1}{3 a} \hat{k}_{y}+\frac{1}{3 c} \hat{k}_{z}\right) .
\end{aligned}
$$

$R \overline{3} c$ is generated ${ }^{14}$ by the elements $\left(E \mid \mathrm{t}_{i}\right)=g_{i}, i=1-3$, $\left(S_{6}^{+} \mid 0\right)=g_{4}$, and $\left(\sigma_{d 1} \mid \tau\right)=g_{5}$, where $\tau=\frac{1}{2} t_{1}+\frac{1}{2} t_{2}+\frac{1}{2} t_{3}$. We noted in the last section that the Lifshitz condition eliminates all irreps associated with $\mathbf{k}$ vectors other than those which are points of symmetry in the holosymmetric point group. These are the $\mathbf{k}$ vectors with centers of symmetry, i.e., with nonpolar point groups. In the trigonal Brillouin zone, with isogonal point group $\overline{3} \mathrm{~m}$, there are four such points, $\Gamma=(000), Z=\left(\frac{1}{2} \frac{1}{2} \frac{1}{2}\right), L=\left(0 \frac{1}{2} 0\right)$ and $F=\left(\frac{1}{2} \frac{1}{2} 0\right)$ (for a $<\sqrt{2} c$ and if $F$ is to be in the first Brillouin zone). ( $x y z)$ are the components of $t$ or $g$, as the case may be, here and below, in the bases of Eqs. (12) or (13).

Among the irreps associated with these four $k$ vectors there are seven active irreps, ${ }^{23}$ i.e., which satisfy the Lifshitz condition and additionally the Landau condition. ${ }^{1}$ Four are associated with the $\Gamma$ point and three with the $F$ point. To find the isotropy groups of $R \overline{3} c$ belonging to these reps we then look at the image subgroups of each rep under the representation homomorphism to find all possible isotropy groups of $L$. The isotropy groups of $G$ are then the inverse images of these. The results for these irreps are listed in Table III.

As an example of how the determination of isotropy subgroups is carried out using this scheme, we consider the irrep $F \Gamma_{2}^{-}$(we use the labeling and notation of Ref. 14). $F \Gamma_{2}^{-}$maps $R \overline{3} c$ onto an image isomorphic to $m 3 m\left(O_{h}\right)$ $=432 \times \overline{1}\left(O \times C_{i}\right)$. The generating elements map as $g_{1} \rightarrow C_{2 y}, g_{2} \rightarrow C_{2 z}, g_{3} \rightarrow C_{2 x}, g_{4}, \rightarrow S_{61}^{+}, g_{5} \rightarrow \sigma_{d e}$. The subgroup hierarchy ${ }^{14}$ of $m 3 m$ is given in Fig. 1 .

Using the well-known characters of $V$, one then finds that $i(4 \mathrm{~mm})=i(4)=i(\mathrm{~mm} 2)=i(3 \mathrm{~m})=i(3)=i(2)=1$, $i(m)=2$, and $i(1)=3$, and $i=0$ for all other subgroups.

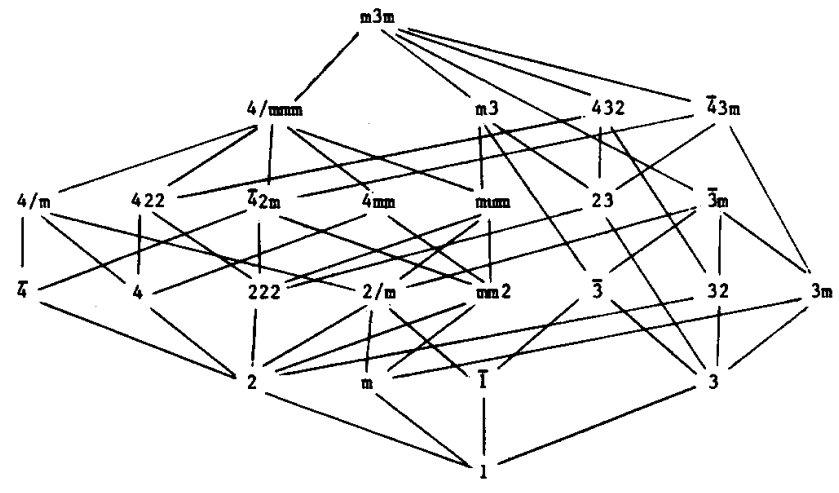

FIG. 1. Subgroups of $m 3 m\left(O_{h}\right)$.

The image subgroup 4 is eliminated by $4 \mathrm{~mm}$ and the chain criterion. $m m 2$ with principle axis in the same direction as that of $4 \mathrm{~mm}$ is eliminated also by the chain criterion, being a subgroup, but $\mathrm{mm} 2$ with principle axis in another direction is not, not being a subgroup. 2 with its axis in the same direction as that of 4 is eliminated, while 2 with axis in the same direction as that of $m m 2$ is also eliminated. The principal axes of 4 are $(x, y, z)$ axes, while the principal axis of $m m 2$ are diagonal $(a, b$, etc.) axes. Finally, the chain criterion eliminates 3 , it being a subgroup of $3 \mathrm{~m}$. We are left with the image isotropy subgroups $4 \mathrm{~mm}, \mathrm{~mm} 2$ (diagonal axis), $3 m, m$, and 1 .

In the representation of $R \overline{3} c$ it is quickly seen that the translations contribute 4 kinds of diagonal matrix images: $\alpha$ with $a_{1}=a_{2}=a_{3}=1, \beta$ with $a_{1}=1, a_{2}=a_{3}$ $=-1, \gamma$ with $a_{1}=a_{3}=-1, a_{2}=1$, and $\delta$ with $a_{1}=a_{2}$ $=-1, a_{3}=1$. Here $a_{i}=\exp \left(i \mathbf{k}_{i} \cdot \mathrm{t}\right)$ with $\mathbf{k}_{i}$ an element of the star of $F$. The actual lattices of the isotropy groups are associated with possible unions of these.

An inverse image of $4 \mathrm{~mm}$ is $\{(E \mid \alpha, \delta),(I \mid \beta, \gamma)$, $\left.\left(C_{22}^{\prime} \mid \beta, \gamma\right),\left(\sigma_{d 2} \mid \alpha, \delta\right)\right\}$. The lattice $\alpha+\delta$ consists of all primitive vectors of the form $\left(n_{1} n_{2} n_{3}\right)$ with $a_{3}=1$, or $n_{1}$ $+n_{3}$ even. This is a primitive monoclinic lattice with doubled unit cell. It has basis vectors $\left(\begin{array}{lll}1 & 0 & 1\end{array}\right),\left(\begin{array}{lll}1 & 0 & \overline{1}\end{array}\right)$, and $(010)$. Call this the (b) lattice. $I$ and $C_{22}^{\prime}$ have an extra translation associated with them, e.g., $t_{1}$. Shifting the origin by $\frac{1}{2} t_{1}$ brings the elements into standard form with both glide planes and screw axes clearly present. The group is thus $P 2_{1} / b(\mathrm{~b})\left(\Gamma_{m} C_{2 h}^{5}\right)$.

The inverse image of $3 m$ is $\{(R \mid \alpha), R \in 3 m\} . \alpha$ is a trigonal lattice with the $n_{i}$ all odd or all even. It is thus essentially a "bcc" lattice on the trigonal basis and has basis vectors $\left(\begin{array}{lll}\overline{1} & 1 & 1\end{array}\right),\left(\begin{array}{lll}1 & \overline{1} & 1\end{array}\right)$, and $\left(\begin{array}{lll}1 & 1 & \overline{1}\end{array}\right)$. Call this lattice (c). The isotropy group is thus $R 3 c$ (c) $\left(\Gamma_{r h} C_{3 v}^{6}\right)$.

The inverse image of $\mathrm{mm} 2$ (diagonal axis) is $\{(E \mid \alpha)$, $\left.(I \mid \delta),\left(C_{22}^{\prime} \mid \delta\right),\left(\sigma_{d 2} \mid \alpha\right)\right\}$. The lattice is the base-centered monoclinic arising from the (c) lattice. It has basis vectors

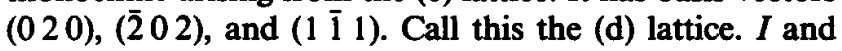
$C_{22}^{\prime}$ have an additional nonprimitive $t^{\prime}$ such that $a_{1}=a_{2}$ $=-1, a_{3}=1$, i.e., $t^{\prime}=t_{2}$. If we shift the axis by $\frac{1}{2} \mathrm{t}_{2},(I \mid 010) \rightarrow(I \mid 000),\left(\sigma_{d 2} \mid \frac{1}{2} \frac{1}{2} \frac{1}{2}\right) \rightarrow\left(\sigma_{d 2} \mid \frac{1}{2} \frac{1}{2} \frac{1}{2}\right)$, and $\left(C_{22}^{\prime}\left(\frac{1}{2} \frac{3}{2} \frac{1}{2}\right) \rightarrow\left(C_{22}^{\prime} \mid \frac{1}{2} \frac{1}{2} \frac{1}{2}\right)\right.$ which clearly shows that this group contains a glide plane, but no screw axis and so must be $C 2 / c(\mathrm{~d})\left(\Gamma_{m}^{b} C_{2 h}^{6}\right)$ or its equivalent. 
One inverse image of $m\left(\left\{E, \sigma_{z}\right\}\right)$ is $\{(E \mid \alpha),(I \mid \delta)\}$ or a triclinic group on the (c) lattice, with displaced origin $\frac{1}{2} t_{2}$, i.e., $P \overline{1}(c)\left(\Gamma_{t} C_{l}^{1}\right)$. Another inverse image is $\{(E \mid \alpha)$, $\left.\left(\sigma_{d} \mid \delta\right)\right\}$, which is $C c\left(\Gamma_{m}^{b} C_{i h}^{4}\right)$, having a glide plane along the new $z$ axis (old $y$ axis) in a base-centered lattice.

Finally the inverse image of 1 is $P 1\left(\Gamma_{t} C_{1}^{1}\right)$, based on the (c) lattice.

We now apply Eqs. (10) and (11). Calcite has two molecules per unit cell of five atoms each. The positions of the atoms in the unit cell, the $d$ labelings, are listed in terms of the basis vectors of Eq. (12) in Table $I$.

Then, in carrying out the sum

$$
\zeta(S)=\sum_{d} e^{-i \mathbf{k} \cdot \mathbf{R}_{d}(S)} \delta\left[\mathbf{d}-S^{-1}(\mathbf{d}-\tau)+\mathbf{R}_{d}(S)\right],
$$

one finds that it is the same for $\mathbf{k}=0$ or $\mathbf{k}=\left(\frac{1}{2} \frac{1}{2} 0\right)$, when looking at the appropriate little group representatives. The results are given in Table II. With this one then finds that the part of $\Gamma^{d}$ belonging to the $\Gamma$ and $F$ point irreps is ${ }^{23,24}$

$$
\begin{aligned}
\Gamma_{\Gamma, F}^{d}= & \Gamma \Gamma_{1}^{+}+3 \Gamma \Gamma_{1}^{-}+2 \Gamma \Gamma_{2}^{+}+4 \Gamma \Gamma_{2}^{-}+4 \Gamma \Gamma_{3}^{+} \\
& +6 \Gamma \Gamma_{3}^{-}+7 F \Gamma_{1}^{-}+10 F \Gamma_{2}^{-}+5 F \Gamma_{1}^{+}+8 F \Gamma_{2}^{+} .
\end{aligned}
$$

Thus, all of the active irreps satisfy the physical field requirement, Eq. (10).

In making the predictions of Table III for the isotropy groups arising out of $R \overline{3} c$ we have used only Eq. (1). When we use also Eq. (4) and minimize it one finds that some of the above isotropy subgroups do not lead to physical phases. Traditionally Landau ${ }^{1}$ only used free energies of the form of Eq. (4) to fourth order in the order parameter components. This is because only fourth order terms are needed to obtain simple symmetry breaking. Indeed, this is all that is needed in the usual $R G$ calculation because of irrelevancy of higher order terms. This, however, does not take into account the full symmetry breaking possibilities of this minimization. Gufan ${ }^{9}$ showed that when the full invariant basis is taken into account for free energies of arbitrary degree, using Eq. (5), the prediction of the nature of the transition may change. In the following both kinds of results are given.

For $\Gamma \Gamma_{1}^{-}, \Gamma \Gamma_{2}^{+}$, and $\Gamma \Gamma_{2}^{-}, L=\overline{1}$, with two matrices, $1,-1$. The Molien function [see Eq. (6)] for these reps is

$$
\frac{1}{2}\left(\frac{1}{1-t}+\frac{1}{1+t}\right)=\frac{1}{1-t^{2}},
$$

from which it is clear that the invariant basis has only one member of order 2 , which must clearly be $x^{2}$ (sub-

TABLE I. Atomic positions in $\mathrm{CaCo}_{3}(\mathrm{I})$. Here $e$ is the distance from the carbon atom to the oxygen atoms in the carbonate $\mathrm{CO}_{3}$ groups.

\begin{tabular}{lll}
\hline \hline $\mathrm{Ca}$ & $(000)$ & $\left(\frac{1}{2} \frac{1}{2} \frac{1}{2}\right)$ \\
$\mathrm{C}$ & $\left(\frac{1}{4} \frac{1}{4} \frac{1}{4}\right)$ & $\left(\frac{3}{4} \frac{3}{4}\right)$ \\
$\mathrm{O}$ & $\left(\frac{1}{4}+e, \frac{1}{4}-e, \frac{1}{4}\right)$ & $\left(\frac{3}{4}-e, \frac{3}{4}+e, \frac{3}{4}\right)$ \\
& $\left(\frac{1}{4}, \frac{1}{4}+e, \frac{1}{4}-e\right)$ & $\left(\frac{3}{4}, \frac{3}{4}-e, \frac{3}{4}+e\right)$ \\
& $\left(\frac{1}{4}-e, \frac{1}{4}, \frac{1}{4}+e\right)$ & $\left(\frac{3}{4}+e, \frac{3}{4}, \frac{3}{4}-e\right)$ \\
\hline \hline
\end{tabular}

TABLE II. Physical characters $\zeta$.

\begin{tabular}{ccccccc}
\hline \hline & $E$ & $I$ & $S_{6}^{ \pm}$ & $C_{3}^{ \pm}$ & $C_{2 i}^{\prime}$ & $\sigma_{d i}$ \\
\hline$\zeta$ & 10 & 2 & 2 & 4 & 4 & 0 \\
\hline \hline
\end{tabular}

stituting $x, y$, etc. for the order parameter components $\sigma_{1}, \sigma_{2}$, etc.).

In these three cases then the free energy takes the form

$$
F=F_{o}+\frac{1}{2} r x^{2}+\frac{1}{24} u x^{4}+\cdots \cdot
$$

Minima occur at $x^{\prime}=0$, when $r>0$, and $x^{\prime}$ $= \pm \sqrt{-6 r / u}$, when $r<0$. Clearly $\rho$ in Eq. (1) is invariant only under those elements of $\overline{3} m$ which are mapped into 1 , corresponding to the isotropy subgroups found earlier (Table III).

Now as $x^{\prime} \rightarrow 0$ one can always maintain $r<0$ so that $x^{\prime}$ remains a minimum of $F$ and we can have a continuous phase transition in these cases. The possible CPT symmetry transitions are $\Gamma \Gamma_{1}^{-}: R \overline{3} c \rightarrow R 32, \Gamma \Gamma_{2}^{+}$: $R \overline{3} c \rightarrow R \overline{3}$ and $\Gamma \Gamma_{2}^{-}: R \overline{3} c \rightarrow R 3 c$. as $^{19}$

The Molien function for $6 \mathrm{~mm}$ is easily calculated

$$
M(t)=\frac{1}{\left(1-t^{2}\right)\left(1-t^{6}\right)},
$$

which indicates that the invariant basis consists of two invariants, one of order 2 and one of order 6 . The sum $\sum_{i} x_{i}^{2}$ is always an invariant, the invariant of order 2 , in this case $x^{2}+y^{2}$. The only possible invariant of order 4 is just this invariant squared, i.e., $\left(\sum_{i} x_{i}^{2}\right)^{2}$. The free energy then has the form

$$
F=\frac{1}{2} r\left(x^{2}+y^{2}\right)+\frac{1}{24} u\left(x^{2}+y^{2}\right)^{2}+\cdots .
$$

In form, this is the same as Eq. (15), if there we substitute $\sigma^{2}=x^{2}+y^{2}$, for $x^{2}$. The minimization then proceeds as before, with extrema at $\sigma=0$ and at $\sigma=\sqrt{-6 r / u}$. The angle is arbitrary, and so we have continuous or degenerate extrema at $\sigma=\sqrt{-6 r / u}$.

The Hessian has eigenvalues $u\left(x^{2}+y^{2}\right) / 6+r$ and $u\left(x^{2}+y^{2}\right) / 2+r$, also clearly independent of the angle. These eigenvalues are $\{r, r\}$ and $\{0,-2 r\}$ respectively for the two types of solutions given above. Clearly the sign of $r$ determines which is a possible minimum.

As already mentioned, a ring of solutions occurs at $\sigma=\sqrt{-6 r / u}$, which is degenerate. Substitution' in Eq. (1) and the use of the matrix forms shows that, however, not all of these points lead to the same isotropy groups. The image isotropy group $m$ occurs for six points, at angles $60^{\circ}$ apart around the origin, as does the image subgroup 2 , while all others on the ring are generic points, or points in the generic stratum, with image isotropy group 1 .

Applying Gufan's results for the image group $6 \mathrm{~mm}$ one finds, however, that the image subgroups $m$ are the only ones that lead to absolute minima, stable in the limit of small order parameter, when the free energy is 
TABLE III. Isotropy groups of the active coreps of $R \overline{3} c$. CPT here indicates that a CPT to the phase indicated can occur along a line of points in $f$ space. SPT indicates that a continuous transition between the phases can occur only at a point. NI indicates that the transition is not indicated to occur at all. NS indicates that the associated extremum of the free energy is nonstable. NSL indicates that while a minimum exists it does not remain stable as $\sigma \rightarrow 0$. (a) Refers to the lattice based on Eqs. (12), (b) has basis vectors (101), (10i), and (010) and is primitive monoclinic. (c) is a trigonal lattice with basis vectors (i11), (1i1), and (11ī). (d) is a base-centered monoclinic lattice with basis vectors (020), (202), and (1ī1).

\begin{tabular}{|c|c|c|c|c|c|c|}
\hline \multirow{2}{*}{$\frac{\text { Irrep }}{\Gamma \Gamma_{1}^{-}}$} & \multirow{2}{*}{$\frac{\text { Image group }}{\overline{1}}$} & \multirow{2}{*}{$\frac{\text { Generator image }}{E, E, E, E, I}$} & \multicolumn{2}{|c|}{ Isotropy subgroup } & \multirow{2}{*}{$\begin{array}{c}\begin{array}{c}\text { Character } \\
\text { in } L_{4}\end{array} \\
\text { CPT }\end{array}$} & \multirow{2}{*}{$\begin{array}{c}\begin{array}{c}\text { Character } \\
\text { in } L_{\infty}\end{array} \\
\text { CPT }\end{array}$} \\
\hline & & & $1 \rightarrow R \overline{\mathbf{3}}$ & (a) & & \\
\hline$\Gamma \Gamma_{2}^{+}$ & $\overline{\mathbf{1}}$ & $E, E, E, I, I$ & $1 \rightarrow R 32$ & (a) & CPT & CPT \\
\hline$\Gamma \Gamma_{2}^{-}$ & $\overline{1}$ & $E, E, E, I, E$ & $1 \rightarrow R 3 c$ & (a) & CPT & CPT \\
\hline$\Gamma \Gamma_{3}^{-}$ & $6 \mathrm{~mm}$ & $E, E, E, \sigma_{d l}, C_{6}^{+}$ & $\begin{array}{l}2 \rightarrow B 2 \\
m \rightarrow B b \\
1 \rightarrow P 1\end{array}$ & $\begin{array}{l}\text { (a) } \\
\text { (a) } \\
\text { (a) }\end{array}$ & $\begin{array}{l}\text { CPT } \\
\text { CPT } \\
\text { CPT }\end{array}$ & $\begin{array}{l}\text { CPT } \\
\text { CPT } \\
\text { NSL }\end{array}$ \\
\hline$F \Gamma_{i}^{-}$ & $m 3 m$ & $C_{2 y}, C_{2 z}, C_{2 x}, S_{61}^{+}, C_{2 e}$ & $\begin{array}{l}3 m \rightarrow R 32 \\
4 m m \rightarrow P 2 / b \\
m m 2 \rightarrow B 2 / b \\
m \rightarrow B 2 \\
m \rightarrow P \overline{1} \\
1 \rightarrow P 1\end{array}$ & $\begin{array}{l}\text { (c) } \\
\text { (b) } \\
\text { (d) } \\
\text { (d) } \\
\text { (c) } \\
\text { (c) }\end{array}$ & $\begin{array}{l}\text { CPT } \\
\text { CPT } \\
\text { NS } \\
\text { NE } \\
\text { NE } \\
\text { NE }\end{array}$ & $\begin{array}{l}\text { CPT } \\
\text { CPT } \\
\text { SPT } \\
\text { SPT } \\
\text { NSL } \\
\text { NSL }\end{array}$ \\
\hline$F \Gamma_{2}^{+}$ & 432 & $C_{2 j}, C_{2 x}, C_{2 z}, C_{31}^{+}, C_{2 e}$ & $\begin{array}{l}3 \rightarrow R \overline{3} \\
4 \rightarrow P 2_{1} / b \\
2 \rightarrow C 2 / c \\
1 \rightarrow P \overline{1}\end{array}$ & $\begin{array}{l}\text { (c) } \\
\text { (b) } \\
\text { (d) } \\
\text { (c) }\end{array}$ & $\begin{array}{l}\text { CPT } \\
\text { CPT } \\
\text { NS } \\
\text { NI }\end{array}$ & $\begin{array}{l}\text { CPT } \\
\text { CPT } \\
\text { SPT } \\
\text { NSL }\end{array}$ \\
\hline$F \Gamma_{2}^{-}$ & $m 3 m$ & $C_{2 y}, C_{2 z}, C_{2 x}, S_{61}^{+}, \sigma_{d e}$ & $\begin{array}{l}3 m \rightarrow R 3 c \\
4 m m \rightarrow P 2_{1} / b \\
m m 2 \rightarrow C 2 / c \\
m \rightarrow C c \\
m \rightarrow P \overline{1} \\
1 \rightarrow P 1\end{array}$ & $\begin{array}{l}\text { (c) } \\
\text { (b) } \\
\text { (d) } \\
\text { (d) } \\
\text { (c) } \\
\text { (c) }\end{array}$ & $\begin{array}{l}\text { CPT } \\
\text { CPT } \\
\text { NS } \\
\text { NI } \\
\text { NI } \\
\text { NI }\end{array}$ & $\begin{array}{l}\text { CPT } \\
\text { CPT } \\
\text { SPT } \\
\text { SPT } \\
\text { NSL } \\
\text { NSL }\end{array}$ \\
\hline
\end{tabular}

extended to arbitrary number of terms. The generic stratum solutions can be made to be minima, just as in the truncated case, but cannot continue to be so, if one requires $\sigma$ to go continuously to 0 for some variation of the parameters $r, u$, etc. Thus, the possible CPT's arising from $\Gamma \Gamma_{3}^{-}$in the classical theory are $R \overline{3} c \rightarrow B 2$ (b) and $R \overline{3} c \rightarrow B b(b)$. $\operatorname{are}^{19}$

The Molien functions for the active $F$ point images

$$
\begin{aligned}
M_{432}(t) & =\frac{1+t^{9}}{\left(1-t^{2}\right)\left(1-t^{4}\right)\left(1-t^{6}\right)}, \\
M_{m 3 m}(t) & =\frac{1}{\left(1-t^{2}\right)\left(1-t^{4}\right)\left(1-t^{6}\right)} .
\end{aligned}
$$
i.e., 9

The invariant bases for $m 3 m$ and 432 are the same,

$$
\begin{aligned}
& I_{1}=x^{2}+y^{2}+z^{2}, \\
& I_{2}=x^{4}+y^{4}+z^{4},
\end{aligned}
$$

and

$$
I_{3}=x^{2} y^{2} z^{2}
$$

$F \mathrm{~T}_{2}^{+}$, as is clear from its Molien function, also has a covariant or relative invariant of ninth order, which is

$$
J_{1}=x y z\left[x^{4}\left(y^{2}-z^{2}\right)+y^{4}\left(z^{2}-x^{2}\right)+z^{4}\left(x^{2}-y^{2}\right)\right] .
$$

To fourth order then, all three active irreps have the same free energy, ${ }^{8}$ e.g.,

$$
\begin{aligned}
F= & \frac{1}{2} r\left(x^{2}+y^{2}+z^{2}\right)+\frac{1}{24} u\left(x^{2}+y^{2}+z^{2}\right)^{2} \\
& +\frac{1}{24} v\left(x^{4}+y^{4}+z^{4}\right),
\end{aligned}
$$

or, in terms of $x_{i}$,

$$
F=\frac{1}{2} r \sum_{i} x_{i}^{2}+\frac{1}{24} u\left(\sum_{i} x_{i}^{2}\right)^{2}+\frac{1}{24} v \sum_{i} x_{i}^{4} .
$$

Extrema occur when

$$
\frac{\partial F}{\partial x_{i}}=r x_{i}+\frac{1}{6} u x_{i} \sigma^{2}+\frac{1}{6} v x_{i}^{3}=0,
$$

and the Hessian matrix has components

$$
\frac{\partial^{2} F}{\partial x_{i} \partial x_{j}}=\left(r+\frac{1}{6} u \sigma^{2}+\frac{1}{2} v x_{i}^{2}\right) \delta_{i j}+\frac{1}{3} u x_{i} x_{j} .
$$

There are four kinds of solutions, or strata, to Eq. (17);

$$
\begin{aligned}
& \text { (1) } \mathrm{x}=0, \\
& \text { (2) } x_{i}=x_{j}=0, \quad x_{j} \neq 0, \quad i \neq j \neq k, \\
& \text { (3) } x_{i}=0, \quad x_{j} \neq 0, \quad i \neq j, \\
& \text { (4) } x_{i} \neq 0 \text { for all } i .
\end{aligned}
$$

Case 1 corresponds to the high symmetry phase and in this phase all the eigenvalues of the Hessian are $r$, and hence it is a minimum when $r>0$.

In case 2, if for example $x \neq 0, y=z=0$, then $x^{2}$ $=\sigma^{2}$ and Eq. (17) leads to $x= \pm \sqrt{-6 r /(u+v)}$ and 
similarly if $y$ or $z \neq 0$ for a total of six points. The eigenvalues of the Hessian are $r v /(u+v)$ (twice) and $-2 r$. A sufficient condition for a strict minimum in this case is thus that $r<0$, and $v /(u+v)<0$. Since $\sigma$ must be real, if $r<0$ then we must have $u+v>0$, which implies that for stability, $v<0$. All these conditions can be maintained as $\sigma \rightarrow 0$, so that these solutions yield viable minima for CPT's, in the truncated theory.

For case 3, if we choose, for definiteness, $x$ and $y$ $>0$ and $z=0$ then it follows that $x^{2}=y^{2}=\frac{1}{2} \sigma^{2}$ and Eq. (17) gives $\sigma=\sqrt{-6 r /(2 u+v)}$. The Hessian eigenvalues are in this case $-2 r v /(2 u+v),-2 r$, and $r v(2 u+v)$. Clearly not all of these can be positive at the same time and so these solutions (of which there are 12) cannot be minima for the truncated free energy.

For case $4, x_{i}, i=1-3$ all satisfy the same equation and so all have the same magnitude. Consider the solution (one of eight) $x=y=z=(1 / \sqrt{3}) \sigma$, such that $\sigma$ $=\sqrt{-6 r /(3 u+v)}$. The Hessian eigenvalues are $-2 r v /(3 u$ $+v$ ) (twice) and $-2 r$. Real, stable solutions will occur if $r<0,3 u+v>0$, and $v>0$. There are no problems in these conditions as $\sigma \rightarrow 0$, if $u$ is kept within a suitable range and so this solution can describe a minimum and a CPT in the truncated theory.

Note that as $v \rightarrow 0$ the above stability conditions fail, in cases 2 and 4 . We then are faced with a situation similar to what happened with $\Gamma \Gamma_{3}^{-}$, when one encounters a ring, or here a sphere, of degenerate solutions. As $v \rightarrow$ 0 then one encounters a first order phase transition from the axis solutions, case 2, to the diagonal solutions, case 4. It is first order since the direction of the order parameter changes abruptly, even though $\sigma$, its magnitude, does not.

For case 2 the density arising from Eq. (1) is, i.e.,

$$
\rho=\sigma \Psi_{1} \text {. }
$$

For case 3 one choice is

$$
\rho=\rho_{0}+\frac{1}{\sqrt{2}} \sigma\left(\Psi_{1}+\Psi_{2}\right)
$$

while for case 4 one choice is

$$
\rho=\rho_{0}+\frac{1}{\sqrt{3}} \sigma\left(\Psi_{1}+\Psi_{2}+\Psi_{3}\right)
$$

For the image group 432 of $F \Gamma_{2}^{+}$, Eqs. (18)-(20) are left invariant by 4,2 , and 3 respectively, corresponding to the inverse images $P 2_{1} / b(\mathrm{~b}), C 2 / c(\mathrm{~d})$, and $R \overline{3}(\mathrm{c})$. The transitions allowed in the truncated theory then by this irrep are two, $R \overline{3} c \rightarrow P 2_{1} / b$ (b) and $R \overline{3} c \rightarrow R \overline{3}$ (c).

For the image group $m 3 m$ of $F \Gamma_{1}^{-}$and $F \Gamma_{2}^{-}$the densities Eqs. (18)-(20) are left invariant by the image groups $4 \mathrm{~mm}, \mathrm{~mm} 2$, and $3 \mathrm{~m}$, respectively, with the corresponding inverse images $P 2 / b(\mathrm{~b}), B 2 / b$, (d), and $R 32$ (c) for $F \Gamma_{1}^{-}$and $P 2_{1} / b(\mathrm{~b}), C 2 / c$ (d), and $R 3 c$ (c) for $F \Gamma_{2}^{-}$. The truncated theory then allows $R \overline{3} c \rightarrow P 2 / b$ (b) or $R 32$ (c) for $F \Gamma_{1}^{-}$and $R \overline{3} c \rightarrow P 2_{1} / b$ (b) or $R 3 c$ (c) for $F \Gamma_{2}^{-}$.

When one uses all possible symmetry information, or rather considers Taylor's series of arbitrary length for the 432 and $m 3 m$ image groups one finds the same results as in the truncated case for 432 , except that the image subgroup 2 of 432 , corresponding to $C 2 / c$ (d) of $F \Gamma_{2}^{+}$is now a possible stable minimum. ${ }^{9}$ It cannot however, produce a CPT, i.e., a line of critical points, since one must impose two conditions on the coefficients $r, u, v$, etc. It could thus describe the symmetry change at an isolated critical point, but not that for a CPT, where there must be only one relevant physical field, i.e., temperature. In addition, a minima occurs in the generic strata, corresponding to the isotropy subgroup of 432 of 1 or the inverse image $P 1$ (c), which cannot both be real, stable and have $\sigma$ go continuously to zero. Hence, this isotropy group cannot lead to a CPT either.

For $m 3 m$ in the general case there are now six types of solutions. In addition to Eqs. (18)-(20) there occur the solutions

$$
\begin{aligned}
& \rho=\rho_{0}+x \Psi_{1}+y \Psi_{2}+z \Psi_{3}, \\
& \rho=\rho_{0}+x \Psi_{1}+y \Psi_{2},
\end{aligned}
$$

and

$$
\rho=\rho_{0}+x\left(\Psi_{1}+\Psi_{2}\right)+y \Psi_{3},
$$

and other solutions of the same symmetry and similar form. Equation (21) belongs to the generic strata and cannot produce a minimum both real and stable as $\sigma \rightarrow$ 0 . Equation (22) has symmetry group $m$ with the reflection plane perpendicular to a major axis and also cannot lead to a minimum both real and stable as $\sigma \rightarrow 0$.

Equation (23) has also the symmetry group $m$, but now with the reflection plane perpendicular to a diagonal axis. It and the solution belonging to Eq. (19), which has symmetry group $m m 2$, can have real, stable continuous transitions, but as before, only at a single point in $f$ space and so cannot describe a CPT either.

The solutions Eqs. (18) and (20) have symmetry groups, respectively, of $3 \mathrm{~m}$ and $4 \mathrm{~mm}$. They both can, under all conditions describe a CPT. The results for $F \Gamma_{1}^{-}$and $F \Gamma_{2}^{-}$, along with those of all other active irreps of $R \overline{3} c$ are summarized in Table III.

We then have carried out a classical mean-field calculation, emphasizing contemporary group action properties, for the possible CPT's arising from the space group $R \overline{3} c$ to all orders in the free energy. The qualitative nature of the complete phase diagram (within mean field)

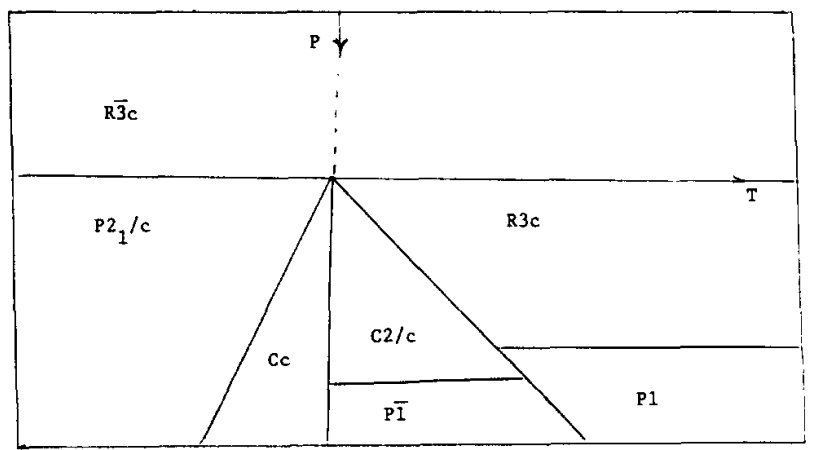

FIG. 2. Qualitative phase diagram of calcite $(R \overline{3} c)$. Phases are indicated by their symmetry group (international notation). 
is thus evident. For example, corresponding to $F \Gamma_{2}^{-}$we obtain $^{9}$ the phase diagram in Fig. 2. The position of the lower symmetry phases $P \overline{1}$ and $P 1$ are only approximate, as are the relative positions of the other phases. The significant feature of the diagram is the behavior of the different phases as $r \rightarrow 0$ [as in Eqs. (15)-(17)], or essentially $T \rightarrow T_{c}$. The phases $P \overline{1}$ and $P 1$ cannot even exist in this region. ${ }^{9}$

' L. D. Landau and E. M. Lifshitz, Statistical Physics (Pergamon, Oxford, 1981).

2 J. W. Felix, Ph.D. dissertation, BYU, Provo, UT, 1984.

${ }^{3}$ John O. Dimmock, Phys. Rev. 130, 1337 (1963).

${ }^{4}$ S. Galam and J. L. Birman, Phys. Lett. A 98, 125 (1983).

${ }^{5}$ The conclusions of the RG are very much based on the assumption of a continuous phase transition and hence large fluctuations near $T_{c}$ and are not applicable to first-order phase transitions in which these never appear.

${ }^{6}$ Reviews can be found in: J. L. Birman, Lect. Notes in Phys. 79, 203 (1978); L. Michel and M. Mozrzymas, ibid. 79, 447 (1978); M. Jaric, Physica A 114, 550 (1982); L. Michel (preprint IHES/P/83/21).

${ }^{7}$ D. M. Hatch and L. M. Merrill, Phys. Rev. B 23, 368 (1981), and D. M. Hatch and D. L. Decker, J. Chem. Phys. 74, 2518 (1981)

${ }^{8}$ V. K. Wadhawhan, Phase Transitions 3, 3 (1982).

${ }^{9}$ Yu M. Gufan and V. P. Sakhnenko, Sov. Phys. JETP 36, 1009 (1973).
${ }^{10}$ P. Curie, J. Phys. (Paris) 5, 197, 263 (1894).

${ }^{11}$ J. L. Birman, Physica A 114, 564 (1982).

${ }^{12}$ For more complete discussions see L. Michel, Rev. Mod. Phys. 52, 617 (1980); M. V. Jarić and M. Senechal (to be published).

${ }^{13}$ M. V. Jarić, Phys. Rev. B 23, 3460 (1981).

${ }^{14} \mathrm{C}$. J. Bradley and A. P. Cracknell, Mathematical Theory of Symmetry in Solids (Oxford University London, 1972).

${ }^{15}$ M. V. Jarić, J. Math. Phys. 24, 2865 (1983); Phys. Rev. Lett. 48, 1641 (1982).

${ }^{16}$ D. M. Hatch, Lect. Notes in Phys. 201, 390 (1984).

${ }^{17}$ D. M. Hatch and H. Stokes, Phys. Rev. B 30, 5156 (1984); H. T. Stokes and D. M. Hatch, Phys. Rev. B 30, 4962 (1984).

${ }^{18}$ R. P. Stanley, Bull. Am. Math. Soc. 1, 475 (1979).

${ }^{19} \mathrm{~J}$. Patera and R. T. Sharp in Recent Advances in Group Theory and their Application to Spectroscopy, edited by J. C. Donini, (Plenum, New York, 1978), p. 219.

${ }^{20} \mathrm{H}$. Brown, R. Bülow, J. Neubüser, H. Wondratschek, and H. Zassenhaus, Crystallographic Groups of Four-Dimensional Space (Wiley, New York, 1978).

${ }^{21}$ J. L. Birman, Lect. Notes in Phys. 79, 203 (1978).

${ }^{22}$ See J. L. Birman, Encyclopedia of Physics (Springer-Verlag, Berlin, 1974), Vol. 25/2b.

${ }^{23}$ D. M. Hatch, Phys. Rev. B 23, 2346 (1981). This reference contains the first calculations of isotropy groups for $R \overline{3} c$ for irreps which satisfy the Landau and Lifshitz conditions. However, we here indicate corrections to Ref. 23.

${ }^{24}$ S. Stornetta and D. M. Hatch, J. Chem. Phys. 80, 5745 (1984). 